\title{
Generation of ultra-high repetition rate pulses in a highly nonlinear dispersion-tailored compound glass fibre
}

\author{
Angela Camerlingo*, Francesca Parmigiani, Radan Slavic, Xian Feng, Francesco Poletti, Peter \\ Horak, Wei H. Loh, Periklis Petropoulos and David J. Richardson \\ Optoelectronics Research Centre, University of Southampton, Southampton SO17 1BJ, United Kingdom \\ *anc@orc.soton.ac.uk
}

\begin{abstract}
The generation of ultrastable optical pulses beyond $160 \mathrm{GHz}$ is demonstrated based on two injection-locked $\mathrm{CW}$ lasers through nonlinear temporal compression in a high SBSthreshold highly nonlinear dispersion tailored compound glass W-type fibre.
\end{abstract}

\section{Introduction}

The generation of high repetition rate pulses through four-wave-mixing (FWM) based nonlinear temporal compression of a dual-frequency beat signal in a highly nonlinear fibre followed by a linear dispersive medium has proven to deliver high quality pedestal-free (sub-) picosecond signals [1-3]. The initially sinusoidal signal can be obtained from the beating of two continuous wave $(\mathrm{CW})$ lasers with a frequency separation corresponding to the desired repetition rate. Usually, independent $\mathrm{CW}$ sources are used [1-2] leading to significant timing jitter on the pulses generated over a timescale inversely proportional to the laser linewidths and which can be a significant issue for many applications. In [3] this issue was addressed using stable injection-locking of two semiconductor lasers to an optical comb, then simultaneously performing narrow-bandwidth filtering and amplification. In that case the two phase-locked lasers were then combined together into 2-m-long highly nonlinear bismuth oxide fibre (Bi-NLF), which has high nonlinear coefficient, but also a high dispersion and dispersion slope. Efficient generation of FWM components generally requires a high nonlinear coefficient and a low dispersion and dispersion slope: much higher repetition rates can be envisaged and much higher pulse compression factors can be achieved as the pulses do not temporally broaden in the nonlinear medium. Compound-glass technology has enabled fibres with much higher values of effective nonlinearity than that achievable in silica. However, compound glasses generally suffer from high normal material dispersion around $1.55 \mu \mathrm{m}$ and counter-balancing this with waveguide dispersion is a non-trivial fabrication task. We have recently shown a fibre design with a W-type refractive index profile as a possible solution to this problem [4] and fabricated a fibre with a highly nonlinear coefficient and low and flat dispersion profile at telecom wavelengths.

Here we experimentally demonstrate the generation of high repetition rate $(160 \mathrm{GHz}$ and $200 \mathrm{GHz})$ pulses through stable injection-locking of two semiconductor lasers in a $3 \mathrm{~m}$-long sample of the fabricated W-type fibre and investigated the role of the tailored dispersion profile for the generation of ultra-short high repetition rate pulses.

\section{Experimental setup and results}

The experimental setup is shown in Fig.1. A CW laser was fed into a $10 \mathrm{GHz}$ spaced optical frequency comb generator (OFCG) [3]. The output of the comb was demultiplexed using a $100 \mathrm{GHz}$ array waveguide grating (AWG) and then coupled into two semiconductor lasers to induce phase locked operation. The combined phase-locked lasers were then amplified and free-space launched into a $3 \mathrm{~m}$ long sample of $\mathrm{W}$-type fibre. The fibre was designed and fabricated to achieve a high nonlinear coefficient and a low dispersion profile at $1550 \mathrm{~nm}$. The fibre core $(1.6 \mu \mathrm{m}$ in diameter) was made of a high index glass, Schott SF57 (n=1.82 @ $1550 \mathrm{~nm})$ which was surrounded by a first cladding $(6.7 \mu \mathrm{m}$ in diameter) made of a low index glass, Schott LLF1 $(\mathrm{n}=1.53 @ 1550 \mathrm{~nm})$. The outer cladding was made of a different glass with a higher refractive index, Schott SF6, (n=1.76 @ 1550nm). The nonlinear coefficient, dispersion and loss at $1550 \mathrm{~nm}$ were $820 \mathrm{~W}^{-1} \mathrm{~km}^{-1},-3 \mathrm{ps} / \mathrm{nm} / \mathrm{km}$ and $2.1 \pm 0.2 \mathrm{~dB} / \mathrm{m}$ respectively [4]. The dispersion profile of the fibre is shown in the inset to Fig. 1. No SBS shift for CW signal powers up to $720 \mathrm{~mW}$ into the fibre was observed. The output end of the fibre was then

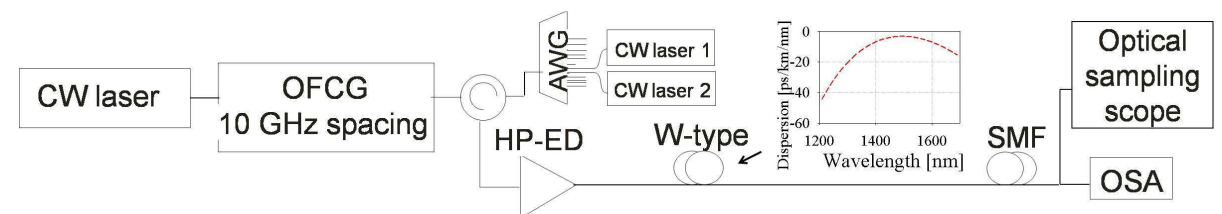

Fig.1: Experimental setup, Inset: dispersion profile of the W-type fibre 

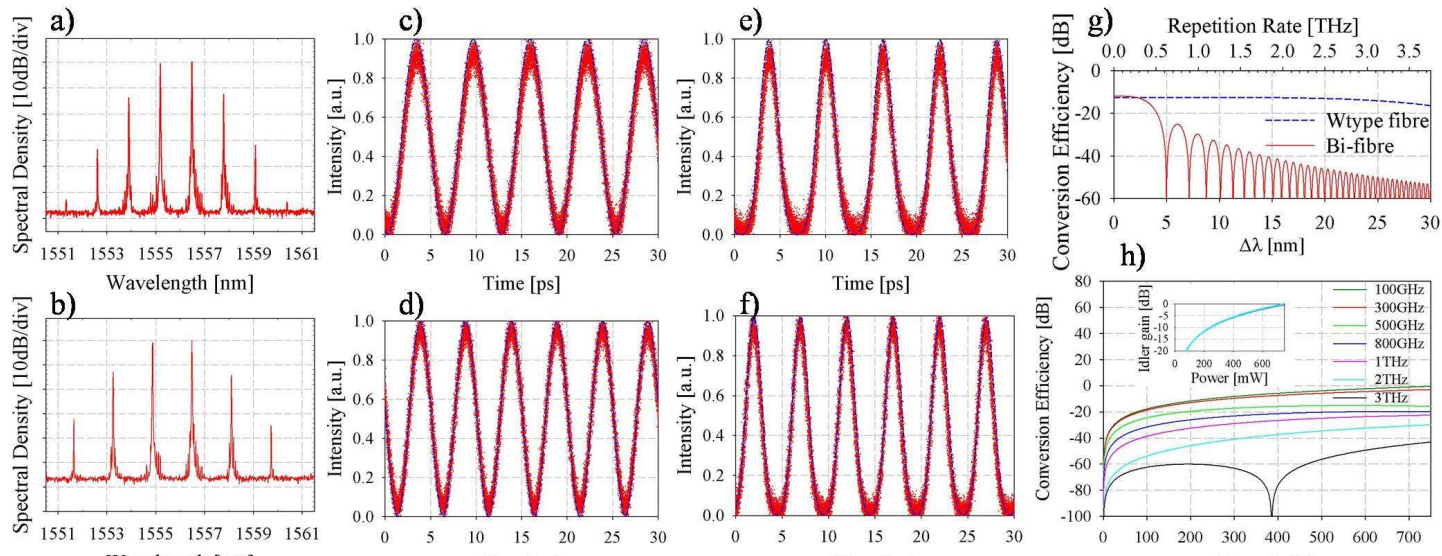

Fig 2: Output spectral trace and temporal traces at the output of the fibre and after the SMF at $160 \mathrm{GHz}$ (a), (c), (e) and at $200 \mathrm{GHz}(\mathrm{b}),(\mathrm{d}),(\mathrm{f})$; Simulated conversion efficiency of the W-type (blue dotted line) and Bi-NLF fibre (red dotted line) for various values of input power and output pulse duration at the output of the W-type fibre (solid blue line) and the Bi-NLF fibre (solid red line) (g), simulated conversion efficiency versus CW spacing for the bismuth fibre (red solid line) and the W-type fibre (blue dotted line) (h).

connected to a few tens of meters of standard single mode fibre (SMF) to temporally compress the pulses. The frequency spacing between the two lasers could be controlled (in $10 \mathrm{GHz}$ steps) by changing the operational temperature of the two phase-locked lasers. The signal in the time and spectral domains at different points along the system are shown in Fig. 2, when the frequency spacing between the two injection-locked lasers was 160 and $200 \mathrm{GHz}$, respectively. Fig. 2(a) and 2(b) show the spectra taken at the output of the W-type fibre where the frequency spacing between the two phaselocked lasers was $160 \mathrm{GHz}$ and $200 \mathrm{GHz}$, respectively. The temporal waveforms were measured using an optical sampling oscilloscope (EXFO Inc.) that was electrically triggered by the OFCG. The initially sinusoidal waves have full-width-at-half-maxima (FWHM) corresponding to a half of the signal period: $3.1 \mathrm{ps}$ and $2.5 \mathrm{ps}$, respectively, for $160 \mathrm{GHz}$ and $200 \mathrm{GHz}$. The pulses at the output of the fibre are shown in Fig.2 (c) and (d), respectively showing negligible temporal broadening as compared to the initial pulses. After the SMF28 the pulses were compressed down to $2.5 \mathrm{ps}(160 \mathrm{GHz})$ and $1.5 \mathrm{ps}(200 \mathrm{GHz})$ respectively, as shown in Fig. 2(e) and 2(f), corresponding to a compression factor of 1.5 and 1.7. These values are comparable to the values of compression factors achieved using the Bi-NLF [3]. In the two experiments indeed, the product $\gamma \mathrm{PL}_{\mathrm{eff}}$, where $\gamma$ is the nonlinear coefficient, $\mathrm{P}$ is the input power and $\mathrm{L}_{\text {eff }}$ is the effective length of the fibres was very comparable. The advantages of the tailored dispersion profile of the W-type fibre can be seen in Fig. 2(g) where the conversion efficiency (the ratio between the first idler and the pump in $\mathrm{dB}$ ) is plotted versus the $\mathrm{CW}$ spacing for the W-type fibre and the Bi-NLF. While the values of conversion efficiency is nearly constant for the W-type fibre (blue dotted line) for any $\mathrm{CW}$ spacing considered, the large value of dispersion of the Bi-NLF has a drastic effect on the conversion efficiency (red solid line) for larger repetition rates. We further investigated the role of the dispersion in the conversion efficiency for values of CW spacing in the range of $100 \mathrm{GHz}$ and $3 \mathrm{THz}$, when increasing the input power. As we can see in Fig. 2(h), the conversion efficiency decreases drastically in the Bi-NLF at higher values of repetition rate, while it remains fairly constant at any of the repetition rates considered for the W-type fibre, as shown in the inset to Fig.2(h).

\section{Conclusions}

We generated short high quality, ultra high repetition rate pulses (beyond $160 \mathrm{GHz}$ ) formed by two phase locked CW lasers and nonlinearly compressed in 3m-long highly nonlinear, dispersion tailored compound glass W-type fibre. The low dispersion and dispersion slope of such fibre play a major role in the generation of short pulses when much higher repetition rates (up to $3 \mathrm{THz}$ ) and higher powers are considered.

\section{Acknowledgments}

The authors would like to thank Eblana Photonics Inc., Dublin, Ireland, for providing the coaxial laser used in the experiment and Dr. A.D. Ellis for useful discussion about injection locking. This research has received funding from the European Communities Seventh Framework Programme FP/2007-2013 under grant agreement 224547 (PHASORS).

\section{References}

[1] S. Pitois, et al., "Generation of a $160 \mathrm{GHz}$ transform-limited pedestal-free pulse train through multi-wave mixing compression of a dual -frequency beat signal", Opt. Lett., 27, 1729-1731, (2002).

[2] T. Inoue, et al., Generation of in-phase pulse train from optical beat signal, Optics Letters 32, 1569 (2007).

[3] F. Parmigiani et al., "Generation of compressed optical pulses beyond $160 \mathrm{GHz}$ based on two injection-locked CW lasers", CLEO/QELS 2010 San Jose 16-21 May 2010.

[4] A. Camerlingo et al., "Wavelength Conversion in a Short Length of a Solid Lead-Silicate Fibre", IEEE Photon. Technology Lett, to appear, 2010. 